European

Thyroid Journal
Eur Thyroid J 2020;9:55-56

DOI: $10.1159 / 000504217$
Received: July 19, 2019

Accepted after revision: October 18, 2019

Published online: November 14, 2019

\title{
Elephantiasic Pretibial Myxedema with Graves' Disease: Spontaneously Induced and Dramatically Resolved after Radioiodine Treatment
}

\author{
Jun Yang Meng-Jie Dong Qin Xu \\ Department of Nuclear Medicine, The First Affiliated Hospital, College of Medicine, Zhejiang University, \\ Hangzhou, PR China
}

\section{Keywords \\ Graves' disease · Pretibial myxedema · Radioiodine}

Pretibial myxedema (PTM) is an uncommon autoimmune manifestation of Graves's disease. It is commonly associated with ophthalmopathy and typically localized in the pretibial region [1]. It is speculated that thyroidstimulating hormone receptor antibody (TRAb) plays an important role in the pathological mechanism. TRAb can trigger an autoimmune response, leading to the inflammatory and infiltrative process with the production of large amounts of hyaluronic acid and glycosaminoglycans in the dermis and subcutaneous tissue [2]. High $\mathrm{TRAb}$ values and antigen release from thyroid cells may induce PTM [3]. Elephantiasic PTM is rare (5\%) [4]. The management of elephantiasic PTM remains a challenge because of the poor patient response and high rate of recurrence.

A 53-year-old Chinese man was admitted to our hospital in December 2012 because he presented a 14-year history of uncontrolled hyperthyroidism. The exophthalmos and dermopathy were not observed. The patient does not smoke. Abnormal laboratory tests included high free thyroxine and free triiodothyronine levels, a mark- edly low thyrotropin titer, thyroperoxidase antibody (TPOAb) level of $>6,500 \mathrm{IU} / \mathrm{mL}$ (reference range, $0-100$ $\mathrm{IU} / \mathrm{mL}$ ), and TRAb level of $>40 \mathrm{IU} / \mathrm{L}$ (reference range, $<1.75 \mathrm{IU} / \mathrm{L}$ ). He received radioiodine treatment $(518$ $\mathrm{MBq}$ ) without steroids. He complained of severe itching and burning of his lower legs and feet after 5 months, and pretibial swelling had gradually increased. During this time, he denied any trauma and maintaining a standing posture for prolonged periods. The patient refused skin biopsy and any treatment for PTM. He was administered a second round of radioiodine $(370 \mathrm{MBq})$ therapy without steroids 8 months later. His TPOAb and TRAb levels were $>6,500 \mathrm{IU} / \mathrm{mL}$ and $>40 \mathrm{IU} / \mathrm{L}$, respectively. However, his lesion progressed to elephantiasic PTM during this time. Cutaneous examination showed multiple bilateral, symmetric, indurated nonpitting verrucous plaques with an orange peel-like appearance on both lower legs and the dorsum of his feet (Fig. 1a). He developed hypothyroidism and subsequently achieved euthyroidism via oral administration of L-euthyroxine for 4 years. Although no additional treatment for PTM was used, his symptoms significantly improved, and the nonpitting edema and all varicose plaques spontaneously disappeared gradually. The skin on his lower legs and dorsum of his feet became smooth and began to appear normal, and only rare hyper-

\section{KARGER}

(C) 2019 European Thyroid Association

Published by S. Karger AG, Basel
Qin Xu

Department of Nuclear Medicine

The First Affiliated Hospital, College of Medicine, Zhejiang University \#79 Qing Chun Road, Hangzhou 310003 (PR China)

E-Mail1187041@zju.edu.cn 
Fig. 1. a Clinical appearance of elephantiasic pretibial myxedema in the patient after radioiodine treatment, revealing multiple indurated nonpitting verrucous plaques with an orange peel-like appearance on his lower legs and dorsum of the feet. $\mathbf{b}$ Anterior appearance of both lower legs and dorsum of the feet without additional treatment, demonstrating that these areas became smooth and appeared normal and that only rare hyperpigmentation remained after 4 years of follow-up.
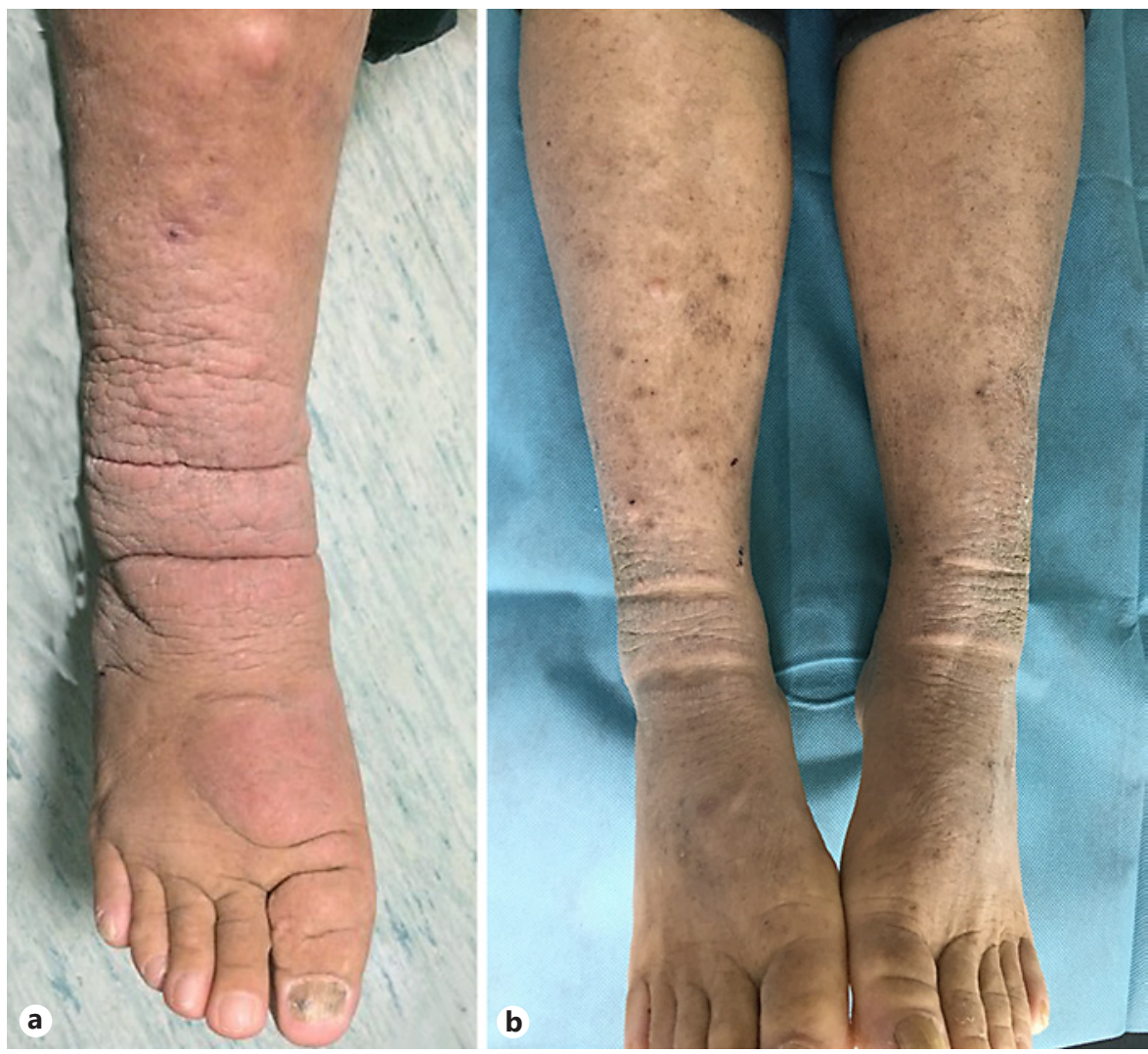

pigmentation remained (Fig. 1b). No recurrence or exophthalmos was observed during the 4 years of follow-up. His TPOAb and TRAb levels gradually decreased to 385 $\mathrm{IU} / \mathrm{mL}$ and $8.35 \mathrm{IU} / \mathrm{L}$, respectively.

\section{Acknowledgement}

We are grateful to Dr. Hongye Fu in the Department of Nuclear Medicine at the First Affiliated Hospital, College of Medicine, Zhejiang University.

\section{Statement of Ethics}

This work was approved by the Ethics Committee of the First Affiliated Hospital, College of Medicine, Zhejiang University. Written informed consent for publication of the clinical details and images was obtained from the patient.

\section{Disclosure Statement}

The authors have no conflicts of interest to declare.

\section{Funding Sources}

This study was supported by the Science and Technology Planning Project of Zhejiang Province, China (2017KY061).

\section{Author Contributions}

Jun Yang and Meng-Jie Dong were involved in acquisition of data and drafting the manuscript. Qin Xu is the corresponding author and organized the study. All authors read and approved the final manuscript.

\section{References}

1 Fatourechi V. Thyroid dermopathy and acropachy. Best Pract Res Clin Endocrinol Metab. 2012 Aug;26(4):553-65.

2 Fatourechi V. Pretibial myxedema: pathophysiology and treatment options. Am J Clin Dermatol. 2005;6(5):295-309.

3 Kriss JP, Pleshakov V, Chien JR. Isolation and identification of the long acting thyroid stimulator and its relation of hyperthyroidism and circumscribed pretibial myxoedema. J Clin Endocrinol Metab. 1964 Oct;24(10):1005-28.

4 Bartalena L, Fatourechi V. Extrathyroidal manifestations of Graves' disease: a 2014 update. J Endocrinol Invest. 2014 Aug;37(8):691-700. 